\title{
The Mysterious Gamma-Ray Excess of Andromeda: Comparing Millisecond Pulsars to Dark Matter
}

\author{
Fabian Zimmer $^{a, *}$ \\ ${ }^{a}$ University of Amsterdam, GRAPPA Institute \\ 1098 XH Amsterdam, Netherlands \\ E-mail: f.zimmer@uva.nl
}

The discovery of an excess in gamma rays coming from the center of our Milky Way galaxy in 2009 has opened up an exciting field in the search for dark matter. More recently in 2017, another such an excess has been confirmed to be present in the Andromeda galaxy. In this work we analyze 10 years of Fermi-LAT data in the attempt to rigorously characterize the significance of this excess when accounting for uncertainties coming from difficulties in modeling the gamma-ray background. For this purpose we constructed specific background models for the Andromeda sky region, employing modern inpainting techniques based on algorithms involving neural networks. We furthermore developed models for testing the hypothesis that this signal could be caused by a population of millisecond pulsars (MSPs), which are as of yet unresolved by the Fermi space telescope. Our findings indicate, that the emission of annihilating dark matter is unwarranted when accounting for the emission of the beforementioned pulsars or contributions from interstellar gas and dust. The results of this work build up on previous attempts on this matter, but we use a more sophisticated and robust statistical framework in combination with templates specifically constructed for this purpose alone.

$37^{\text {th }}$ International Cosmic Ray Conference (ICRC 2021)

July 12th-23rd, 2021

Online - Berlin, Germany

\footnotetext{
*Presenter
} 


\section{Introduction}

The discovery of the excess in gamma rays coming from the center of the Andromeda galaxy in 2017 [2] led to a multitude of studies on this phenomenon. It appears to be coming from the inner regions of the galaxy, i.e. the bulge but has more recently been observed to extend even further outwards [6]. Like the similar gamma-ray excess of the Milky Way center, the cause of this excess is still not completely understood. There is mounting evidence however, that both could be caused by unresolved MSP populations in the bulge of the galaxies. Such pulsars have been shown to be able to accelerate particles, like electrons and positrons, to high enough energies, such that they can produce gamma rays through secondary processes.

We have conducted a dedicated analysis to test whether the MSP hypothesis is preferred over the more exotic explanation involving emission from dark matter annihilation in the center of the galaxy. To this end, we have constructed stellar density maps, specifically designed for this region of the sky, which we use to trace such a MSP population. We furthermore investigated, how moddeling of the diffuse gamma-ray background influences the significance of this excess. We both build upon and improve on previous studies such as for example [4] and [3].

We analysed 10 years of Fermi-Lat data, restricting ourselves to a dataset with more reliable reconstructed directions of the measured photons, which make conclusions about the morphology more robust. We employed a bin-by-bin analysis method and therefore divided the data into 10 logarithmically spaced energy bins from $500 \mathrm{MeV}$ to $100 \mathrm{GeV}$. In each energy bin we assume the underlying spectral shape to be a simple power law with a fixed spectral slope of -2 , such that there is no bias in the fitting procedure due to the assumption of a specific spectral shape.
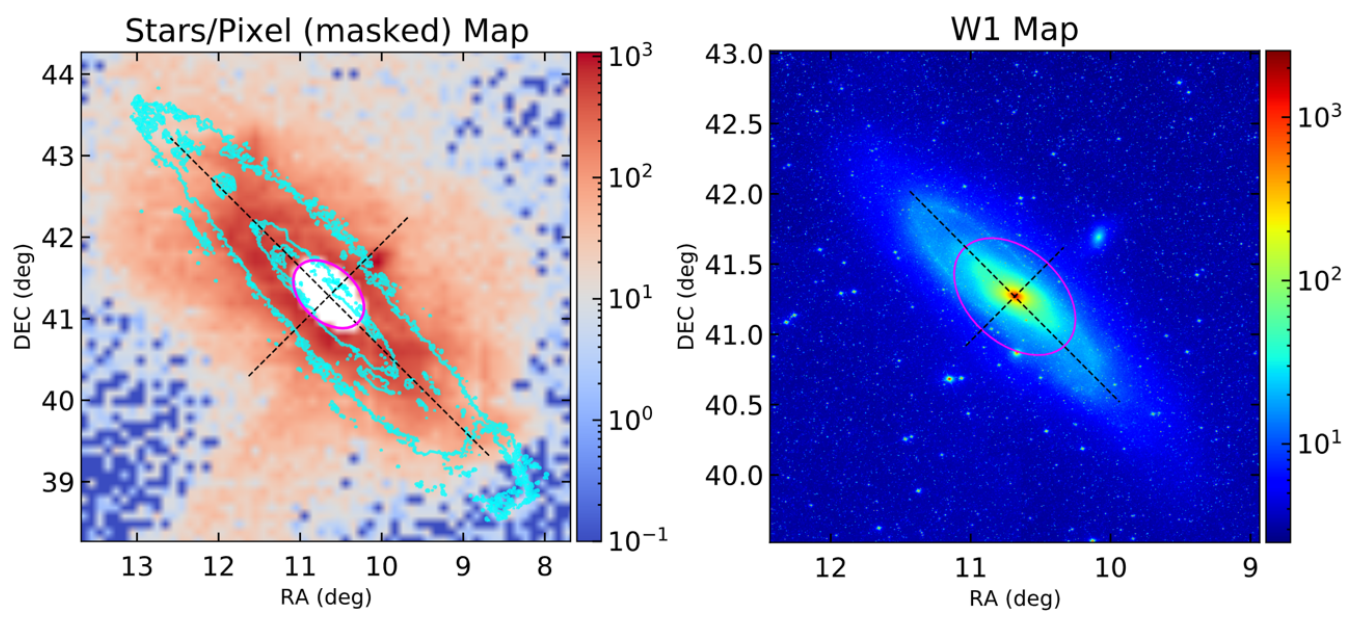

Figure 1: Left: An old red giant population from the PAndAS archive which we used for the disk component of the stellar density map, where we masked the center because the data was unreliable in that region. Right: Infrared observations from WISE, which we used for the bulge component of the stellar density map. The magenta ellipses have the same spatial dimensions. 


\section{Millisecond Pulsars and Dark Matter}

To approximate the spatial distribution of the MSPs in the bulge of Andromeda, we used observational data of old red giants. The theory behind the formation of a star towards a MSP predicts, that the timescale is comparable to the lifetime of a red giant. Therefore we can use old stellar populations, which are bright enough to be observed in contrast to the unobserved MSPs, to get a sense of where these pulsars might be. We used a combination of data from the PAndAS [5] and WISE [8] to build our stellar density maps. In Fig. 1 we see the density of these old stars from these archives. We use the stellar data inside the magenta ellipse in the right panel to replace the unreliable data in the magenta ellipse in the left panel.

For our dark matter template we model the density with the Navarro-Frenk-White (NFW) profile, which reads

$$
\rho(r)=\frac{\rho_{0}}{\frac{r}{r_{s}}\left(1+\frac{r}{r_{s}}\right)^{2}},
$$

where $\rho_{0}$ and $r_{s}$ are halo dependent parameters. This density is squared and itegrated along the line-of-sight towards Andromeda to get the J-factor as

$$
J_{p}(l, b)=\int_{s} \rho(r[s, l, b])^{2} d s .
$$

\section{Background Uncertainties}

Both previously mentioned studies ([4] and [3]) have used different methods to get to the systematic uncertainties coming with the gamma-ray background modelling. In this work, we have constructed alternative diffuse gamma-ray background templates using a variety of novel inpainting techniques. We have used different hydrogen tracing maps, based on hydrodynamical simulations as described in [7] together with alternative inverse Compton templates from [1]. Combining all this together we arrive at a total of

$$
2(\mathrm{H} 1 \text { components }) \times 3(\text { Inpainting Methods }) \times 4(\text { Inverse Compton Models })=24
$$

alternative diffuse background models.

\section{Discussion and conclusions}

With this dedicated study of the Andromeda gamma-ray excess we arrive at three main conclusions.

1. When we include additional components, such as our stellar disk or bulge components, in the background model, we find that the addition of a dark matter components quickly becomes unwarranted as can be seen in Fig. 2

2. In the case for our dataset, which has less statistical power but more reliable reconstructed direction of the photons, we find that the flux uncertainties are dominated by errors of statistical rathern than systematic origin and all our alternative background models yield comparable flux values. 


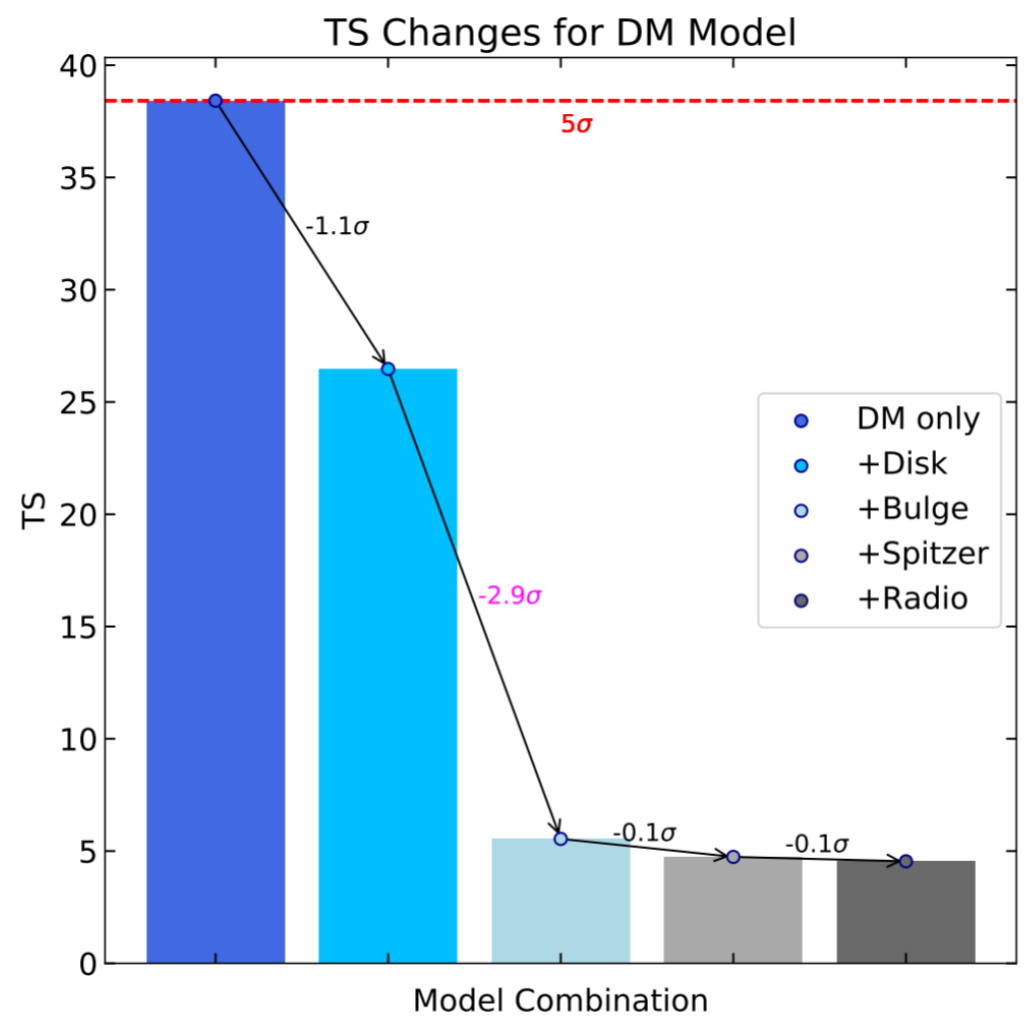

Figure 2: The significance in terms of the test statistic (TS, as the likelihood ratio of null to alternative hypothesis) of the dark matter component, when we step-wise incorporate additional templates into the background model, i.e. the null hypothesis.

3. Our findings seem to indicate, that the emission is not spatially correlated with gas and dust sites in the galaxy but rather with the stellar mass in the bulge of Andromeda. This further strengthens the hypothesis, that the emission could be due to an unresolved population of millisecond pulsars in the bulge of Andromeda.

\section{References}

[1] M. Ackermann et al. The spectrum of isotropic diffuse gamma-ray emission between $100 \mathrm{MeV}$ and $820 \mathrm{GeV}$. Astrophys. J., 799:86, 2015.

[2] M. Ackermann et al. Observations of M31 and M33 with the Fermi Large Area Telescope: A Galactic Center Excess in Andromeda? Astrophys. J., 836(2):208, 2017.

[3] Céline Armand and Francesca Calore. Gamma-ray image reconstruction of the Andromeda galaxy. Phys. Rev. D, 103(8):083023, 2021.

[4] Mattia Di Mauro, Xian Hou, Christopher Eckner, Gabrijela Zaharijas, and Eric Charles. Search for $\gamma$-ray emission from dark matter particle interactions from Andromeda and Triangulum Galaxies with the Fermi Large Area Telescope. Phys. Rev. D, 99(12):123027, 2019. 
[5] Rodrigo A. Ibata et al. The Large-scale Structure of the Halo of the Andromeda Galaxy. I. Global Stellar Density, Morphology and Metallicity Properties. Astrophys. J., 780:128, 2014.

[6] Chris Karwin, Simona Murgia, Sheldon Campbell, and Igor Moskalenko. Fermi-LAT Observations of $\gamma$-Ray Emission Towards the Outer Halo of M31. PoS, ICRC2019:570, 2021.

[7] Oscar Macias, Chris Gordon, Roland M. Crocker, Brendan Coleman, Dylan Paterson, Shunsaku Horiuchi, and Martin Pohl. Galactic bulge preferred over dark matter for the Galactic centre gamma-ray excess. Nature Astron., 2(5):387-392, 2018.

[8] Edward L. Wright, Peter R. M. Eisenhardt, Amy K. Mainzer, Michael E. Ressler, Roc M. Cutri, Thomas Jarrett, J. Davy Kirkpatrick, Deborah Padgett, Robert S. McMillan, Michael Skrutskie, and et al. The wide-field infrared survey explorer (wise): Mission description and initial on-orbit performance. The Astronomical Journal, 140(6):1868-1881, Nov 2010. 